ELECTRONIC RESEARCH ANNOUNCEMENTS OF THE AMERICAN MATHEMATICAL SOCIETY

Volume 12, Pages 29-36 (March 6, 2006)

S $1079-6762(06) 00157-0$

\title{
STRONG LAWS FOR WEIGHTED SUMS OF I.I.D. RANDOM VARIABLES
}

\author{
GUANG-HUI CAI
}

(Communicated by Mark Freidlin)

\begin{abstract}
Strong laws are established for linear statistics that are weighted sums of a random sample. We show extensions of the Marcinkiewicz-Zygmund strong laws under certain moment conditions on both the weights and the distribution. The result obtained extends and sharpens the result of Sung.
\end{abstract}

\section{INTRODUCTION}

Many useful linear statistics based on a random sample are weighted sums of independent and identically distributed (i.i.d.) random variables. Examples include least-squares estimates, nonparametric regression function estimates, and jackknife estimates, among others. In this respect, studies of strong laws for these weighted sums have demonstrated significant progress in probability theory with applications in mathematical statistics. Let $\left\{X_{i}, i \geq 1\right\}$ be i.i.d. copies of a random variable $X$, and $\left\{a_{n i}, 1 \leq i \leq n, n \geq 1\right\}$ a triangular array of constants. The almost sure (a.s.) limiting behavior of weighted sums $\sum_{i=1}^{n} a_{n i} X_{i}$ was studied by many authors (see [1, 2, 4, 9, 10]). Recently, Sung (see [9]) proved the following strong laws of large numbers.

Theorem 1.1. Let $\left\{X, X_{i}, i \geq 1\right\}$ be a sequence of i.i.d. random variables satisfying $E X=0$, and for any $h>0$ and some $\gamma>0, E \exp \left(h|X|^{\gamma}\right)<\infty$. Let $\left\{a_{n i}, 1 \leq i \leq\right.$ $n, n \geq 1\}$ be a triangular array of constants satisfying

$$
A_{\alpha}=\limsup _{n \rightarrow \infty} A_{\alpha, n}<\infty,
$$

where $A_{\alpha, n}=\sum_{i=1}^{n}\left|a_{n i}\right|^{\alpha} / n$ for some $1<\alpha \leq 2$. Then for $0<\gamma<1$ and $b_{n}=n^{\frac{1}{\alpha}}(\log n)^{\frac{1}{\gamma}}$,

$$
\sum_{i=1}^{n} a_{n i} X_{i} / b_{n} \rightarrow 0 \text { a.s.; }
$$

moreover, for $\gamma>1$ and $b_{n}=n^{\frac{1}{\alpha}}(\log n)^{\frac{1}{\gamma}+\delta}$,

$$
\sum_{i=1}^{n} a_{n i} X_{i} / b_{n} \rightarrow 0 \text { a.s. }
$$

Received by the editors February 24, 2005 and, in revised form, October 17, 2005.

2000 Mathematics Subject Classification. Primary 60F15.

Key words and phrases. Almost sure convergence, Marcinkiewicz-Zygmund strong laws.

The author would like to thank an anonymous referee for his/her valuable comments.

(C)2006 American Mathematical Society 
Theorem 1.2. Let $\left\{X, X_{i}, i \geq 1\right\}$ be a sequence of i.i.d. random variables satisfying $E X=0$, and for some $h, \gamma>0, E \exp \left(h|X|^{\gamma}\right)<\infty$. Let $\left\{a_{n i}, 1 \leq i \leq n, n \geq 1\right\}$ be a triangular array of constants satisfying $A_{\alpha}=\lim _{\sup _{n \rightarrow \infty}} A_{\alpha, n}<\infty$, where $A_{\alpha, n}=\sum_{i=1}^{n}\left|a_{n i}\right|^{\alpha} / n$ for some $1<\alpha \leq 2$. Then for $0<\gamma \leq 1$ and $b_{n}=$ $n^{\frac{1}{\alpha}}(\log n)^{\frac{1}{\gamma}+\beta}$, for any $\beta>0$,

$$
\sum_{i=1}^{+n} a_{n i} X_{i} / b_{n} \rightarrow 0 \text { a.s.; }
$$

moreover, for $\gamma>1$ and $b_{n}=n^{\frac{1}{\alpha}}(\log n)^{\frac{1}{\gamma}+\delta+\beta}$, for any $\beta>0$

$$
\sum_{i=1}^{n} a_{n i} X_{i} / b_{n} \rightarrow 0 \quad \text { a.s. }
$$

where $\delta=1-\frac{1}{\gamma}-\frac{\gamma-1}{1+\alpha \gamma-\alpha}>0$.

The main purpose of this paper is to establish the Marcinkiewicz-Zygmund strong laws for linear statistics of i.i.d. sequences of random variables. The results obtained (see Theorem 2.3 and Corollary 2.16) extend and sharpen the result of Sung (see [9]). As a corollary of our Theorem 2.3, $b_{n}$ in Theorems 1.1 and 1.2 can be taken as $b_{n}=n^{\frac{1}{\alpha}}(\log n)^{\frac{1}{\gamma}}$ for $0<\alpha \leq 2$ thus improving and extending the Sung result.

\section{The MarcinkiewicZ-Zygmund Strong LAWS}

Throughout this paper, $C$ will represent a positive constant, though its value may change from one appearance to the next, and $a_{n}=O\left(b_{n}\right)$ will mean $a_{n} \leq C b_{n}$. In order to prove our results, we need the following lemma and the concept of complete convergence.

Definition 2.1 (see [6]). Let $\left\{X, X_{n}, n \geq 1\right\}$ be a sequence of random variables. If for any $\varepsilon>0$,

$$
\sum_{n=1}^{\infty} P\left(\left|X_{n}-X\right|>\varepsilon\right)<\infty
$$

holds, we call $\left\{X_{n}, n \geq 1\right\}$ completely converging to $X$.

Now let $\left\{X, X_{n}, n \geq 1\right\}$ be a sequence of independent identically distributed random variables, and denote $S_{n}=\sum_{i=1}^{n} X_{i}$. The Hsu-Robbins-Erdös law of large numbers (proved first in [6] and [5]) states that the following conditions are equivalent:

(1) $\forall \varepsilon>0, \sum_{n=1}^{\infty} P\left(\left|S_{n}\right|>\varepsilon n\right)<\infty$;

(2) $E X=0$ and $E X^{2}<\infty$.

This is a fundamental theorem in probability theory, and it has been intensively investigated by many authors in the past decades. We can see it in [7, 3] and [8]. There have been many extensions in various directions of the Hsu-Robbins-Erdös law of large numbers.

Lemma 2.2 (see [7, page 84]). Let $\left\{X_{i}, i \geq 1\right\}$ be a sequence of independent random variables, $E X_{i}=0, E\left|X_{i}\right|^{p}<\infty$ for some $p \geq 2$ and for every $i \geq 1$. Then there 
exists $C=C(p)$ such that

$$
E \max _{1 \leq k \leq n}\left|\sum_{i=1}^{k} X_{i}\right|^{p} \leq C\left\{\sum_{i=1}^{n} E\left|X_{i}\right|^{p}+\left(\sum_{i=1}^{n} E X_{i}^{2}\right)^{p / 2}\right\} .
$$

Theorem 2.3. Let $\left\{X, X_{i}, i \geq 1\right\}$ be a sequence of i.i.d. random variables. Let $\left\{a_{n i}, 1 \leq i \leq n, n \geq 1\right\}$ be a triangular array of constants satisfying $A_{\alpha}=$ $\lim \sup _{n \rightarrow \infty} A_{\alpha, n}<\infty, A_{\alpha, n}=\sum_{i=1}^{n}\left|a_{n i}\right|^{\alpha} / n$ for some $1<\alpha \leq 2$. Let $T_{n}=$ $\sum_{i=1}^{n} a_{n i} X_{i}, n \geq 1, b_{n}=n^{\frac{1}{\alpha}}(\log n)^{\frac{1}{\gamma}}$. For $1<\alpha \leq 2$, it is assumed that $E X=0$. We also assume that for some $h, \gamma>0, E \exp \left(h|X|^{\gamma}\right)<\infty$. Then for any $\varepsilon>0$,

$$
\sum_{n=1}^{\infty} n^{-1} P\left(\max _{1 \leq j \leq n}\left|T_{j}\right|>\varepsilon b_{n}\right)<\infty
$$

Proof. For any $i \geq 1$, define $X_{i}^{(n)}=X_{i} I\left(\left|X_{i}\right| \leq b_{n}\right)$ and

$$
T_{j}^{(n)}=\sum_{i=1}^{j}\left(a_{n i} X_{i}^{(n)}-E a_{n i} X_{i}^{(n)}\right) .
$$

Then for any $\varepsilon>0$,

$$
\begin{aligned}
& P\left(\max _{1 \leq j \leq n}\left|T_{j}\right|>\varepsilon b_{n}\right) \\
& \quad \leq P\left(\max _{1 \leq j \leq n}\left|X_{j}\right|>b_{n}\right)+P\left(\max _{1 \leq j \leq n}\left|T_{j}^{(n)}+\sum_{i=1}^{j} E a_{n i} X_{i}^{(n)}\right|>\varepsilon b_{n}\right) \\
& \quad \leq P\left(\max _{1 \leq j \leq n}\left|X_{j}\right|>b_{n}\right)+P\left(\max _{1 \leq j \leq n}\left|T_{j}^{(n)}\right|>\varepsilon b_{n}-\max _{1 \leq j \leq n}\left|\sum_{i=1}^{j} E a_{n i} X_{i}^{(n)}\right|\right) .
\end{aligned}
$$

First we show that

$$
b_{n}^{-1} \max _{1 \leq j \leq n}\left|\sum_{i=1}^{j} E a_{n i} X_{i}^{(n)}\right| \rightarrow 0 \text { as } n \rightarrow \infty .
$$

From the assumptions of the theorem we know that $A_{\alpha}=\lim \sup _{n \rightarrow \infty} A_{\alpha, n}<\infty$ and $A_{\alpha, n}=\sum_{i=1}^{n}\left|a_{n i}\right|^{\alpha} / n$ for some $1<\alpha \leq 2$. Then we have

$$
\sum_{i=1}^{n}\left|a_{n i}\right|^{\alpha}=O(n)
$$

From the fact that $\sum_{i=1}^{n}\left|a_{n i}\right|^{\alpha}=O(n)$ and Hölder's inequality, it follows that for any $1 \leq k<\alpha$,

$$
\sum_{i=1}^{n}\left|a_{n i}\right|^{k} \leq\left(\sum_{i=1}^{n}\left|a_{n i}\right|^{k \frac{\alpha}{k}}\right)^{\frac{k}{\alpha}}\left(\sum_{i=1}^{n} 1\right)^{\frac{\alpha-k}{\alpha}} \leq C n .
$$

When $1<\alpha \leq 2$, we can use that $E X=0$, inequality (2.7), Markov's inequality, 
and the fact that $E \exp \left(h|X|^{\gamma}\right)<\infty$ as $n \rightarrow \infty$, to prove the following:

$$
\begin{aligned}
b_{n}^{-1} \max _{1 \leq j \leq n}\left|\sum_{i=1}^{j} E a_{n i} X_{i}^{(n)}\right| & \leq b_{n}^{-1} \sum_{i=1}^{n} E\left|a_{n i} X_{i}\right| I\left(\left|X_{i}\right|>b_{n}\right) \\
& =b_{n}^{-1} \sum_{i=1}^{n}\left|a_{n i}\right| E|X| I\left(|X|>b_{n}\right) \\
& \leq C b_{n}^{-1} n E|X| I\left(|X|>b_{n}\right) \\
& =C b_{n}^{-1} n \sum_{k=n}^{\infty} E|X| I\left(b_{k}<|X| \leq b_{k+1}\right) \\
& \leq C b_{n}^{-1} n \sum_{k=n}^{\infty} b_{k+1} P\left(|X|>b_{k}\right) \\
& \leq C b_{n}^{-1} n \sum_{k=n}^{\infty} b_{k+1} \frac{E \exp \left(h|X|^{\gamma}\right)}{\exp \left(h b_{k}^{\gamma}\right)} \\
& \leq C b_{n}^{-1} n \sum_{k=n}^{\infty}(k+1)^{\frac{1}{\alpha}}(\log (k+1))^{\frac{1}{\gamma}} k^{-h k^{\gamma / \alpha}} \\
& \leq C n^{-\frac{1}{\alpha}}(\log n)^{-\frac{1}{\gamma}} n n^{-1} \\
& =C n^{-\frac{1}{\alpha}}(\log n)^{-\frac{1}{\gamma}} \rightarrow 0 .
\end{aligned}
$$

From the fact that $\sum_{i=1}^{n}\left|a_{n i}\right|^{\alpha}=O(n)$ and Hölder's inequality, it follows that for any $k \geq \alpha$,

$$
\sum_{i=1}^{n}\left|a_{n i}\right|^{k}=\sum_{i=1}^{n}\left|a_{n i}\right|^{\alpha}\left|a_{n i}\right|^{k-\alpha} \leq C n n^{\frac{k-\alpha}{\alpha}}=C n^{\frac{k}{\alpha}} .
$$

When $0<\alpha \leq 1$, we can use inequality (2.9), Markov's inequality, and the fact that $E \exp \left(h|X|^{\gamma}\right)<\infty$ as $n \rightarrow \infty$, to prove the following:

$$
\begin{aligned}
b_{n}^{-1} \max _{1 \leq j \leq n}\left|\sum_{i=1}^{j} E a_{n i} X_{i}^{(n)}\right| & \leq b_{n}^{-1} \sum_{i=1}^{n} E\left|a_{n i} X_{i}\right| I\left(\left|X_{i}\right| \leq b_{n}\right) \\
& =b_{n}^{-1} \sum_{i=1}^{n}\left|a_{n i}\right| E|X| I\left(|X| \leq b_{n}\right) \\
& \leq C b_{n}^{-1} n^{\frac{1}{\alpha}} E|X| I\left(|X| \leq b_{n}\right) \\
& =C b_{n}^{-1} n^{\frac{1}{\alpha}} \sum_{k=2}^{n} E|X| I\left(b_{k-1}<|X| \leq b_{k}\right) \\
& \leq C b_{n}^{-1} n^{\frac{1}{\alpha}} \sum_{k=2}^{n} b_{k} P\left(|X|>b_{k-1}\right) \\
& \leq C b_{n}^{-1} n^{\frac{1}{\alpha}} \sum_{k=2}^{n} b_{k} \frac{E \exp \left(h|X|^{\gamma}\right)}{\exp \left(h b_{k-1}^{\gamma}\right)} \\
& \leq C b_{n}^{-1} n^{\frac{1}{\alpha}} \sum_{k=2}^{n} k^{\frac{1}{\alpha}}(\log k)^{\frac{1}{\gamma}}(k-1)^{-h(k-1)^{\gamma / \alpha}} \\
& \leq C n^{-\frac{1}{\alpha}}(\log n)^{-\frac{1}{\gamma}} n^{\frac{1}{\alpha}} \\
& =C(\log n)^{-\frac{1}{\gamma}} \rightarrow 0 .
\end{aligned}
$$


We see that (2.6) follows from (2.8) and (2.10).

We can continue by noticing that for $n$ large enough, it follows from (2.5) and (2.6) that

$$
P\left(\max _{1 \leq j \leq n}\left|T_{j}\right|>\varepsilon b_{n}\right) \leq \sum_{j=1}^{n} P\left(\left|X_{j}\right|>b_{n}\right)+P\left(\max _{1 \leq j \leq n}\left|T_{j}^{(n)}\right|>\frac{\varepsilon}{2} b_{n}\right) .
$$

Hence we need only to prove that

$$
\begin{aligned}
I & =: \sum_{n=1}^{\infty} n^{-1} \sum_{j=1}^{n} P\left(\left|X_{j}\right|>b_{n}\right)<\infty, \\
I I & =: \sum_{n=1}^{\infty} n^{-1} P\left(\max _{1 \leq j \leq n}\left|T_{j}^{(n)}\right|>\frac{\varepsilon}{2} b_{n}\right)<\infty .
\end{aligned}
$$

From the fact that $E \exp \left(h|X|^{\gamma}\right)<\infty$, it easily follows that

$$
\begin{aligned}
I & =\sum_{n=1}^{\infty} n^{-1} n P\left(|X|>b_{n}\right) \\
& =\sum_{n=1}^{\infty} P\left(|X|>b_{n}\right) \\
& \leq \sum_{n=1}^{\infty} \frac{E \exp \left(h|X|^{\gamma}\right)}{\exp \left(h b_{n}^{\gamma}\right)} \\
& \leq C \sum_{n=1}^{\infty} \frac{1}{n^{h n^{\gamma / \alpha}}}<\infty .
\end{aligned}
$$

From Lemma 2.1, it follows that

$$
\begin{aligned}
I I & \leq C \sum_{n=2}^{\infty} n^{-1} b_{n}^{-q} E \max _{1 \leq j \leq n}\left|T_{j}^{(n)}\right|^{q} \\
& \leq C \sum_{n=2}^{\infty} n^{-1} b_{n}^{-q}\left\{\sum_{j=1}^{n} E\left|a_{n j} X_{j}^{(n)}\right|^{q}+\left(\sum_{j=1}^{n} E\left|a_{n j} X_{j}^{(n)}\right|^{2}\right)^{q / 2}\right\} \\
& =: I I_{1}+I I_{2} .
\end{aligned}
$$


Let $\max (2, \alpha, \gamma+1) \leq q$; using (2.9), we have

$$
\begin{aligned}
I I_{1} & =C \sum_{n=2}^{\infty} n^{-1} b_{n}^{-q} \sum_{i=1}^{n}\left|a_{n i}\right|^{q} E|X|^{q} I\left(|X| \leq b_{n}\right) \\
& \leq C \sum_{n=2}^{\infty} n^{-1} b_{n}^{-q} n^{\frac{q}{\alpha}} E|X|^{q} I\left(|X| \leq b_{n}\right) \\
& =C \sum_{n=2}^{\infty} n^{-1} b_{n}^{-q} n^{\frac{q}{\alpha}} \sum_{k=2}^{n} E|X|^{q} I\left(b_{k-1}<|X| \leq b_{k}\right) \\
& \leq C \sum_{k=2}^{\infty} \sum_{n=k}^{\infty} n^{-1+\frac{q}{\alpha}} n^{-q / \alpha}(\log n)^{-q / \gamma} b_{k}^{q} P\left(|X|>b_{k-1}\right) \\
& \leq C \sum_{k=2}^{\infty} b_{k}^{q} \frac{E \exp \left(h|X|^{\gamma}\right)}{\exp \left(h b_{k-1}^{\gamma}\right)} \\
& \leq C \sum_{k=2}^{\infty} k^{\frac{q}{\alpha}}(\log k)^{\frac{q}{\gamma}}(k-1)^{-h(k-1)^{\gamma / \alpha}}<\infty .
\end{aligned}
$$

Using $0<\alpha \leq 2$, (2.9), and $q \geq \max \{2, \gamma+1\}$, we see that

$$
\begin{aligned}
I I_{2} & =C \sum_{n=2}^{\infty} n^{-1} b_{n}^{-q}\left(\sum_{i=1}^{n}\left|a_{n i}\right|^{2}\right)^{q / 2}\left(E|X|^{2} I\left(|X| \leq b_{n}\right)\right)^{q / 2} \\
& \leq C \sum_{n=2}^{\infty} n^{-1} b_{n}^{-q}\left(n^{\frac{2}{\alpha}}\right)^{q / 2}\left(E|X|^{2} I\left(|X| \leq b_{n}\right)\right)^{q / 2} \\
& =C \sum_{n=2}^{\infty} n^{-1}(\log n)^{-q / \gamma}\left(\sum_{k=2}^{n} E|X|^{2} I\left(b_{k-1}<|X| \leq b_{k}\right)\right)^{q / 2} \\
& \leq C \sum_{n=2}^{\infty} n^{-1}(\log n)^{-q / \gamma}\left(\sum_{k=2}^{n} b_{k}^{2} P\left(|X|>b_{k-1}\right)\right)^{q / 2} \\
& \leq C \sum_{n=2}^{\infty} n^{-1}(\log n)^{-q / \gamma}\left(\sum_{k=2}^{n} b_{k}^{2} \frac{E \exp \left(h|X|^{\gamma}\right)}{\exp \left(h b_{k-1}^{\gamma}\right)}\right)^{q / 2} \\
& \leq C \sum_{n=2}^{\infty} n^{-1}(\log n)^{-q / \gamma}\left(\sum_{k=2}^{n} \frac{k^{\frac{2}{\alpha}}(\log k)^{\frac{2}{\gamma}}}{\exp \left(h(k-1)^{\gamma / \alpha} \log (k-1)\right)}\right)^{q / 2} \\
& =C \sum_{n=2}^{\infty} n^{-1}(\log n)^{-q / \gamma}\left(\sum_{k=2}^{n} k^{\frac{2}{\alpha}}(\log k)^{\frac{2}{\gamma}}(k-1)^{-h(k-1)^{\gamma / \alpha}}\right)^{q / 2} \\
& \leq C \sum_{n=2}^{\infty} n^{-1}(\log n)^{-q / \gamma}\left(\sum_{k=2}^{n} k^{-2}\right)^{q / 2} \\
& \leq C \sum_{n=2}^{\infty} n^{-1}(\log n)^{-q / \gamma}<\infty .
\end{aligned}
$$

Putting (2.14) and (2.15) into (2.13) yields $I I<\infty$. This completes the proof of Theorem 2.3 . 
Corollary 2.16. Under the assumptions of Theorem 2.3.

$$
\lim _{n \rightarrow \infty} \frac{\left|T_{n}\right|}{b_{n}}=0 \text { a.s. }
$$

Proof. By (2.4), we have

$$
\begin{aligned}
\infty & >\sum_{n=1}^{\infty} n^{-1} P\left(\max _{1 \leq j \leq n}\left|T_{j}\right|>\varepsilon b_{n}\right) \\
& =\sum_{i=0}^{\infty} \sum_{n=2^{i}}^{2^{i+1}-1} n^{-1} P\left(\max _{1 \leq j \leq n}\left|T_{j}\right|>\varepsilon n^{\frac{1}{\alpha}}(\log n)^{\frac{1}{\gamma}}\right) \\
& \geq \frac{1}{2} \sum_{i=1}^{\infty} P\left(\max _{1 \leq j \leq 2^{i}}\left|T_{j}\right|>\varepsilon 2^{\frac{i+1}{\alpha}}\left(\log 2^{i+1}\right)^{\frac{1}{\gamma}}\right) .
\end{aligned}
$$

By the Borel-Cantelli lemma, we have

$$
P\left(\max _{1 \leq j \leq 2^{i}}\left|T_{j}\right|>\varepsilon 2^{\frac{i+1}{\alpha}}\left(\log 2^{i+1}\right)^{\frac{1}{\gamma}} \text { for infinitely many } i\right)=0 .
$$

Hence

Using

$$
\lim _{i \rightarrow \infty} \frac{\max _{1 \leq j \leq 2^{i}}\left|T_{j}\right|}{2^{\frac{i+1}{\alpha}}\left(\log 2^{i+1}\right)^{\frac{1}{\gamma}}}=0 \quad \text { a.s. }
$$

we have

$$
\max _{2^{i-1} \leq n<2^{i}} \frac{\left|T_{n}\right|}{b_{n}} \leq 2^{\frac{2}{\alpha}} \frac{\max _{1 \leq j \leq 2^{i}}\left|T_{j}\right|}{2^{\frac{i+1}{\alpha}}\left(\log 2^{i+1}\right)^{\frac{1}{\gamma}}}\left(\frac{i+1}{i-1}\right)^{\frac{1}{\gamma}}
$$

$$
\lim _{n \rightarrow \infty} \frac{\left|T_{n}\right|}{b_{n}}=0 \text { a.s. }
$$

Remark 2.17. As a corollary of our Theorem 2.3, $b_{n}$ in Theorems 1.1 and 1.2 can be taken as $b_{n}=n^{\frac{1}{\alpha}}(\log n)^{\frac{1}{\gamma}}$ for $0<\alpha \leq 2$, thus improving and extending the result by Sung obtained in [9].

\section{REFERENCES}

[1] Bai, Z. D. and Cheng, P. E., Marcinkiewicz strong laws for linear statistics, Statist. Probab. Lett. 46 (2000), 105-112. MR1748864 (2001b:60043)

[2] Choi, B. D. and Sung, S. H., Almost sure convergence theorems of weighted sums of random variables, Stochastic Anal. Appl. 5 (1987), 365-377. MR0912863 (88m:60079)

[3] Chow, Y. S. and Teicher, H., Probability Theory: Independence, Interchangeability, Martingales, Springer-Verlag, New York, 3rd ed., 1997. MR.1476912 (98e:60003)

[4] Cuzick, J., A strong law for weighted sums of i.i.d. random variables, J. Theoret. Probab. 8 (1997), 625-641. MR.1340830 (96f:60050)

[5] Erdös, P., On a theorem of Hsu-Robbins, Ann. Math. Statist. 20 (1949), 286-291. MR0030714 $(11,40 f)$

[6] Hsu, P. L. and Robbins, H., Complete convergence and the law of large numbers, Proc. Nat. Acad. Sci. (USA) 33 (1947), 25-31. MR0019852 (8,470e)

[7] Petrov, V. V., Limit theorems of probability theory sequences of independent random variables, Oxford, Oxford Science Publications, 1995. MR1353441 (96h:60048)

[8] Stout, W., Almost sure convergence, New York, Academic Press, 1974. MR0455094 (56 \#13334) 
[9] Sung, S. H., Strong laws for weighted sums of i.i.d. random variables, Statist. Probab. Lett. 52 (2001), 413-419. MR1841609 (2002f:60054)

[10] Wu, W. B., On the strong convergence of a weighted sum, Statist. Probab. Lett. 44 (1999), 19-22. MR.1706378 (2000d:60057)

Department of Mathematics and Statistics, Zhejiang Gongshang University, HangZHOU 310035, P. R. CHINA

E-mail address: cghzju@163.com 